\title{
Analysis of bifidobacterial evolution using a multilocus approach
}

\author{
Marco Ventura, ${ }^{1,2}$ Carlos Canchaya, ${ }^{1}$ Antonio Del Casale, ${ }^{3}$ \\ Franco Dellaglio, ${ }^{3}$ Erasmo Neviani, ${ }^{2}$ Gerald F. Fitzgerald ${ }^{1}$ \\ and Douwe van Sinderen ${ }^{1}$
}

\begin{abstract}
Correspondence
Marco Ventura

marco.ventura@unipr.it
\end{abstract}

\author{
${ }^{1}$ Alimentary Pharmabiotic Centre and Department of Microbiology, Bioscience Institute, National \\ University of Ireland, Western Road, Cork, Ireland \\ ${ }^{2}$ Department of Genetics, Anthropology and Evolution, University of Parma, Parco Area delle \\ Scienze 11a, 43100 Parma, Italy \\ ${ }^{3}$ Dipartimento Scientifico e Tecnologico, University of Verona, Italy
}

\begin{abstract}
Bifidobacteria represent one of the most numerous groups of bacteria found in the gastrointestinal tract of humans and animals. In man, gastrointestinal bifidobacteria are associated with health effects and for this reason they are often used as functional ingredients in food and pharmaceutical products. Such applications may benefit from or require a clear and reliable bifidobacterial species identification. The increasing number of available bacterial genome sequences has provided a large amount of housekeeping gene sequences that can be used both for identification of bifidobacterial species as well as for understanding bifidobacterial evolution. In order to assess their relative positions in the evolutionary process, fragments from seven conserved genes, $c / p C, d n a B, d n a G$, $d n a J 1$, purF, rpoC and $x f p$, were sequenced from each of the currently described type strains of the genus Bifidobacterium. The results demonstrate that the concatenation of these seven gene sequences for phylogenetic purposes allows a significant increase in the discriminatory power between taxa.
\end{abstract}

\section{INTRODUCTION}

Bifidobacteria represent one of the largest bacterial groups within the Actinobacteria. Currently, over 30 species of the genus Bifidobacterium are recognized. Bifidobacteria have been isolated from a number of environments such as sewage (Scardovi \& Trovatelli, 1974), an anaerobic digester (Dong et al., 2000) and fermented milk (Meile et al., 1997; Masco et al., 2004), but their common, and probably natural, habitat is considered to be the intestine of man and other warm-blooded mammals (for a review see Ventura et al., 2004b). In the human gastrointestinal tract, their presence has been associated with beneficial health effects, such as immunomodulation, amelioration of lactose intolerance and prevention of diarrhoea. Thus, some members of the genus have been used as prophylactic and

\footnotetext{
Abbreviations: COG, clusters of orthologous groups; HGT, horizontal gene transfer.

The GenBank/EMBL/DDBJ accession numbers for the partial 16S rRNA, clpC, dnaB, dnaG, dnaJ1, purF, rpoC and xfp gene sequences generated in this study are shown in the phylogenetic trees in Supplementary Figs S1-S8.

Additional phylogenetic trees of the genus Bifidobacterium generated using $c / p C, d n a J 1, r p o C$, purF, dnaB, dnaG, xfp and groEL gene sequences are available as supplementary figures in IJSEM Online.
}

therapeutic agents for human health (for a review see Ouwehand et al., 2002). Due to the growing industrial importance of and associated scientific interest in bifidobacteria, it has become increasingly important to establish a precise classification scheme for the increasing number of (sometimes closely related) bifidobacterial species.

During the last decade, the development of molecular biological tools has led to profound modifications in the identification methodologies of these bacteria and has resulted in various classification adjustments. Most currently available bifidobacterial identification tools, such as amplified rDNA restriction analysis (ARDRA; Ventura et al., 2001a), species-specific PCR primers (Matsuki et al., 1999; Ventura et al., 2001b) and denaturing gradient gel electrophoresis (DGGE; Favier et al., 2002), are based on $16 S$ rRNA gene sequence analysis. However, within the genus Bifidobacterium, the interspecies similarity of this sequence is relatively high, ranging from $87 \cdot 7$ to $99 \cdot 5 \%$. Some bifidobacterial taxa have a very high degree of similarity or even possess identical 16S rRNA gene sequences, such as Bifidobacterium animalis subsp. animalis and Bifidobacterium animalis subsp. lactis, Bifidobacterium longum biotype longum, Bifidobacterium longum biotype infantis and Bifidobacterium longum biotype suis, Bifidobacterium coryneforme, Bifidobacterium asteroides 
and Bifidobacterium indicum, and Bifidobacterium catenulatum and Bifidobacterium pseudocatenulatum. In recent years, alternative molecular markers have been proposed for bifidobacterial identification, such as groEL (Jian et al., 2001; Ventura \& Zink, 2003; Ventura et al., 2004c), recA (Kullen et al., 1997; Ventura \& Zink, 2003), tuf (Ventura \& Zink, 2003), atpD (Ventura et al., 2004a), dnaK (Ventura et al., 2005a) and grpE (Ventura et al., 2005a). However, there is currently no complete sequence database available for such genes and these molecules do not fulfil all the criteria in order to be considered as suitable evolutionary clocks (Ludwig \& Schleifer, 1999). Molecular methods using a single gene are popular because they yield quick and unequivocal results, but in some cases suffer from the disadvantage that they do not generate sufficient discriminatory power. The combined use of several genes for detailed classification purposes, also called the multigenic approach, complies with the recent recommendations of the ad hoc committee for the re-evaluation of the bacterial species definition (Stackebrandt et al., 2002). It recommends that, for the description of a novel species, sequences from four or five housekeeping genes besides the 16S rRNA gene are taken into account. Based on the above, we developed a multigene sequence database dedicated to the identification and discrimination of species and subspecies within the genus Bifidobacterium. We obtained the partial sequence of seven genes, clpC, dnaB, dnaG, dnaJ1, purF, rpoC and $x f p$, for each type strain of the currently described bifidobacterial species. In this report, we analyse the phylogenetic results, compare the phylogenetic trees resulting from these data and show the significance of the use of more than one gene in phylogenetic reconstruction. Finally, we demonstrate how the concatenation of various conserved genes allows an increase in discriminatory power to provide a more robust phylogenetic tree.

\section{METHODS}

Bacterial strains and culture conditions. All strains used in this study are type strains, except for Bifidobacterium breve UCC 2003. All Bifidobacterium strains were grown anaerobically in MRS (Difco) supplemented with $0.05 \%$ L-cysteine- $\mathrm{HCl}$ and incubated at $37^{\circ} \mathrm{C}$ for $16 \mathrm{~h}$.

DNA extraction and gene amplification. Chromosomal DNA was extracted as described previously (Ventura et al., 2001a). PCR was used to amplify segments of $c l p C, d n a B, d n a G, p u r F$, rpoC and dnaJ1, correlating to fragment sizes of 561, 207, 372, 450, 690 and $537 \mathrm{bp}$, using primer combinations ClpC-uni and ClpC-rev, DnaBuni and DnaB-rev, DnaG-uni and DnaG-rev, PurF-uni and PurFrev, RpoC-uni and RpoC-rev, DnaJ1-uni and DnaJ1-rev, respectively (see Table 1 for sequence details). These primers were based on positions 2457-3018 and 99-639 of the published $c l p C$ and dnaJ1 gene sequences, respectively, from B. breve UCC 2003 (Ventura et al., 2005b, c) and positions 765-972, 408-780, 609-1059 and 1194-1884 of the publicly available sequences of the predicted $d n a B, d n a G$, purF and rpoC genes, respectively, from Bifidobacterium longum biotype longum DJO10A (GenBank). Moreover, in order to complete the existing Bifidobacterium sequence database for the $x f p$ gene (Yin et al., 2005), a 120 bp PCR amplicon was amplified using the primer
Table 1. Oligonucleotides used in this study

\begin{tabular}{|ll|}
\hline Oligonucleotide & \multicolumn{1}{c|}{ Sequence $\left(\mathbf{5}^{\prime} \rightarrow \mathbf{3}^{\prime}\right)$} \\
\hline DnaJ1-uni & GAGAAGTTCAAGGACATCTC \\
DnaJ1-rev & GCTTGCCCTTGCCGG \\
PurF-uni & CATTCGAACTCCGACACCGA \\
PurF-rev & GTGGGGTAGTCGCCGTTG \\
RpoC-uni & GTGCACTCGGTCCACAG \\
RpoC-rev & CATGCTCAACAACGAGAAG \\
DnaB-uni & GTGCTTGGCCATGATGAT \\
DnaB-rev & ACCTCGACAAGGTCGG \\
DnaG-uni & CTGTGCCCGTTCCACGAC \\
DnaG-rev & CTCGATGCGCAGGTCGCA \\
Xfp-uni & CTTCGG(AGCT)CC(AGCT)GA(AGCT)GAGAC \\
Xfp-rev & AGCCACTT(AGCT)GC(AGCT)TGCTG \\
ClpC-uni & GAGTACCGCAAGTACATCGAG \\
ClpC-rev & CATCCTCATCGTCGAACAGGAAC \\
\hline
\end{tabular}

pair Xfp-uni and Xfp-rev (Table 1) targeting positions 2342-2462 of the published $x f p$ gene sequence of Bifidobacterium animalis subsp. lactis DSM $10140^{\mathrm{T}}$ (Meile et al., 2001).

PCR were carried out in $50 \mu \mathrm{l}$ volumes containing $20 \mathrm{mM}$ Tris/ $\mathrm{HCl}$, $50 \mathrm{mM} \mathrm{KCl}, 200 \mu \mathrm{M}$ each deoxynucleoside triphosphate, $50 \mathrm{pmol}$ each primer, $1.5 \mathrm{mM} \mathrm{MgCl}_{2}$ and $1 \mathrm{U}$ Taq DNA polymerase (Gibco$\mathrm{BRL}$ ). Each PCR cycling profile consisted of an initial denaturation step of $5 \mathrm{~min}$ at $95^{\circ} \mathrm{C}$, followed by amplification for 35 cycles as follows: denaturation $\left(30 \mathrm{~s}\right.$ at $\left.95^{\circ} \mathrm{C}\right)$, annealing $\left(30 \mathrm{~s}\right.$ at $\left.51^{\circ} \mathrm{C}\right)$ and extension $\left(1 \mathrm{~min}\right.$ at $\left.72^{\circ} \mathrm{C}\right)$. The PCR was completed with an elongation phase $\left(10 \mathrm{~min}\right.$ at $\left.72{ }^{\circ} \mathrm{C}\right)$. The resulting amplicons were separated on a $0 \cdot 8 \%$ agarose gel, followed by ethidium bromide staining. PCR fragments were purified using a PCR purification spin kit (Qiagen) and were subsequently sequenced.

No amplification products representing $\operatorname{clp} C$, purF, dnaG or dnaJ1 could be obtained for a small number of strains even though alternative primer combinations and various experimental conditions were used (data not shown).

The genome sequences of $B$. longum biotype longum DJO10A were used as sources for the DNA sequences of $c l p C, d n a B, d n a G, d n a J 1$, $p u r F, r p o C$ and $x f p$ gene sequences. Thus PCRs were not performed for this strain.

Sequence analysis. Sequence data were edited using SeqMan software from the DNASTAR package (http://www.dnastar.com). Sequences were aligned using CLUSTAL W (Thompson et al., 1994). Phylogenetic analysis and trees, which were based on the derived amino acid sequences of the obtained DNA sequences, were calculated using PHYLIP, version 3.5c (Felsenstein, 1993) and NJplot (Perrière \& Gouy, 1996). Trees were calculated using the neighbourjoining method under the Kimura's two-parameter model as the substitution model (Kimura, 1980). Phylogenetic trees were also calculated by the maximum-likelihood method with PHYML software (Guindon \& Gascuel, 2003), which allows a discrete-gamma model to be used to accommodate rate variation among sites. Bootstrap values of the supertree were computed by resampling 100 times.

Slot-blot hybridization. A $15 \mu \mathrm{g}$ sample of bacterial DNA was spotted onto a Hybond-N + membrane (Amersham) using a BioDot SF micro-filtration apparatus (Bio-Rad), as specified by the manufacturer, and treated with one UV-autocross-linking cycle using a UV Stratalinker 1800 (Stratagene). Filters were hybridized 
with a $B$. breve- $B$. suis- $B$. infantis- $B$. animalis subsp. animalis- $B$. catenulatum-B. asteroides-derived $c l p C, d n a G, d n a J 1$ and purF probe mix, respectively, which was labelled with $\left[\alpha{ }^{32} \mathrm{P}\right]$ using the random primed DNA labelling system (Roche). Each probe mix was obtained by mixing amplicons obtained from DNA extracted from the above mentioned species. Subsequent prehybridization, hybridization and autoradiography were carried out according to Sambrook et al. (2001).

Nucleotide accession numbers. The GenBank accession numbers for the partial $16 \mathrm{~S}$ rRNA, clpC, dnaB, dnaG, dnaJ1, purF, rpoC and $x f p$ gene sequences used and generated in this study are indicated in each tree.

\section{RESULTS AND DISCUSSION}

\section{Phylogenetic analysis}

A preliminary screening of the current actinobacterial genomes for conserved genes (C. Canchaya, M. Ventura, G. Fitzgerald, and D. van Sinderen, unpublished results) revealed the presence of 143 conserved genes which may represent the minimal gene set for this group of bacteria. However, some of the genes considered highly conserved by this approach might be acquired by transposition or by horizontal gene transfer (HGT) events and it is reasonable to assume that increasing the number of actinobacterial, or indeed bifidobacterial, genomes used in the comparison would significantly reduce the number of such essential genes. A first criterion used to select genes suitable for our study was to eliminate those genes from this minimal actinobacterial gene set that were predicted to be affected by HGT events as evaluated by $\mathrm{G}+\mathrm{C}$ skew, dinucleotide frequency and codon usage analyses. Furthermore, a preliminary evaluation of DNA sequence conservation extended to all currently recognized bifidobacterial species using a PCR approach was considered to be an indispensable tool to identify conserved genes. Seven genes, $c l p C$, $d n a B$, dnaG, dnaJ1, purF, rpoC and xfp, fulfilled most of our requirements and consequently were used to investigate the phylogeny of the currently described bifidobacterial species. Gene fragments of the seven genes were obtained from 32 strains representing the type strain of each species of the genus Bifidobacterium. Despite several unsuccessful efforts using different sets of PCR primers and PCR amplification parameters (data not shown), fragments could not be obtained for the following: the $c l p C$ gene from Bifidobacterium minimum LMG $11592^{\mathrm{T}}$, the purF gene from Bifidobacterium saeculare LMG $14934^{\mathrm{T}}$, the $d n a G$ genes from Bifidobacterium angulatum ATCC $27535^{\mathrm{T}}$ and $B$. thermacidophilum subsp. thermacidophilum LMG $21395^{\mathrm{T}}$ and the dnaJ1 genes from Bifidobacterium indicum LMG $11587^{\mathrm{T}}$, B. angulatum ATCC 27535 and Bifidobacterium merycicum LMG $11341^{\mathrm{T}}$. This failure is possibly be due to DNA sequence variability in these genes which apparently prevented annealing of the PCR primers to their respective targeting sites. In all of the other 217 cases, a sequence could be obtained for every gene for each of the 32 strains. For each gene, a tree was calculated in order to evaluate the overall compatibility between the different trees. In addition, a phylogenetic tree was constructed using the 16S rRNA gene sequences available in databases for the same set of bifidobacterial strains (Fig. 1). Clusters supported by bootstrap values greater than $80 \%$ in the seven phylogenetic trees were identified (Fig. 1). Species included within clusters supported by strong bootstrap values in one of these trees are generally associated in the same strong clusters in other trees or are not associated in another cluster supported by high bootstrap values. The use of two alternative treecreating methods, neighbour-joining method and maximum-likelihood, revealed very similar tree topologies (data not shown). Although an overall similarity is evident in all gene trees for some species (e.g. Bifidobacterium bifidum, Bifidobacterium scardovii and Bifidobacterium subtile), as also noted in a previous report (Dellaglio \& Felis, 2005), the phylogenetic positioning is not clearly defined. However, given the different phylogenetic information content of the different molecular markers, missing monophyly in part of the trees or failure of significant assignment of certain branches, as indicated above, to clusters in some or all trees shows different resolution powers which are not expected to create discrepancies if a combined phylogenetic analysis is performed.

Seven phylogenetic trees were produced using the individual genes and an additional tree was created on the basis of the concatenation of these sequences.

\section{Single-gene trees}

A phylogenetic analysis based on the $16 \mathrm{~S}$ rRNA gene sequences of the type strains of the 34 currently recognized species, subspecies or biotypes of the genus Bifidobacterium was performed. This analysis resulted in a phylogenetic tree (Fig. 1) that is consistent with previously described bifidobacterial taxonomic analyses (Miyake et al., 1998; Ventura et al., 2004b). The $16 \mathrm{~S}$ rRNA gene allows the discrimination of most species within the genus Bifidobacterium. Analysis of bifidobacterial phylogeny based on $16 \mathrm{~S}$ rRNA gene sequences shows incongruence between the ecological origin of bifidobacterial taxa and their evolution. In fact, except for the bifidobacterial group isolated from insects, i.e. $B$. asteroides-B. indicum and $B$. coryneforme, which cluster together, the remainder of the bifidobacterial ecological groups, i.e. human, bovine, pig and chicken isolates, were not obviously linked on the basis of their $16 \mathrm{~S}$ rRNA gene sequence relatedness. However, the tree based on the 16S rRNA gene sequences is robust. Of the nodes, $46 \%$ are supported by bootstrap values greater than $80 \%$. Sequence similarities among all the Bifidobacterium taxa range from $87 \cdot 7$ to $99 \cdot 5 \%$ (mean $95 \%$ ) and are exceptionally high for the following groups: Bifidobacterium pseudolongum subsp. pseudolongum and Bifidobacterium pseudolongum subsp. globosum group (similarity 99.3-99.9\%), B. animalis subsp. animalis-B. animalis subsp. lactis group (similarity $99 \%$ ), B. catenulatum and Bifidobacterium pseudocatenulatum group (similarity $99 \cdot 5 \%$ ), B. indicum-B. asteroides group (similarity 99.1\%) and $B$. longum biotype longum, $B$. longum biotype suis and $B$. longum biotype infantis (similarity $99 \cdot 1-99 \cdot 2 \%$ ). 


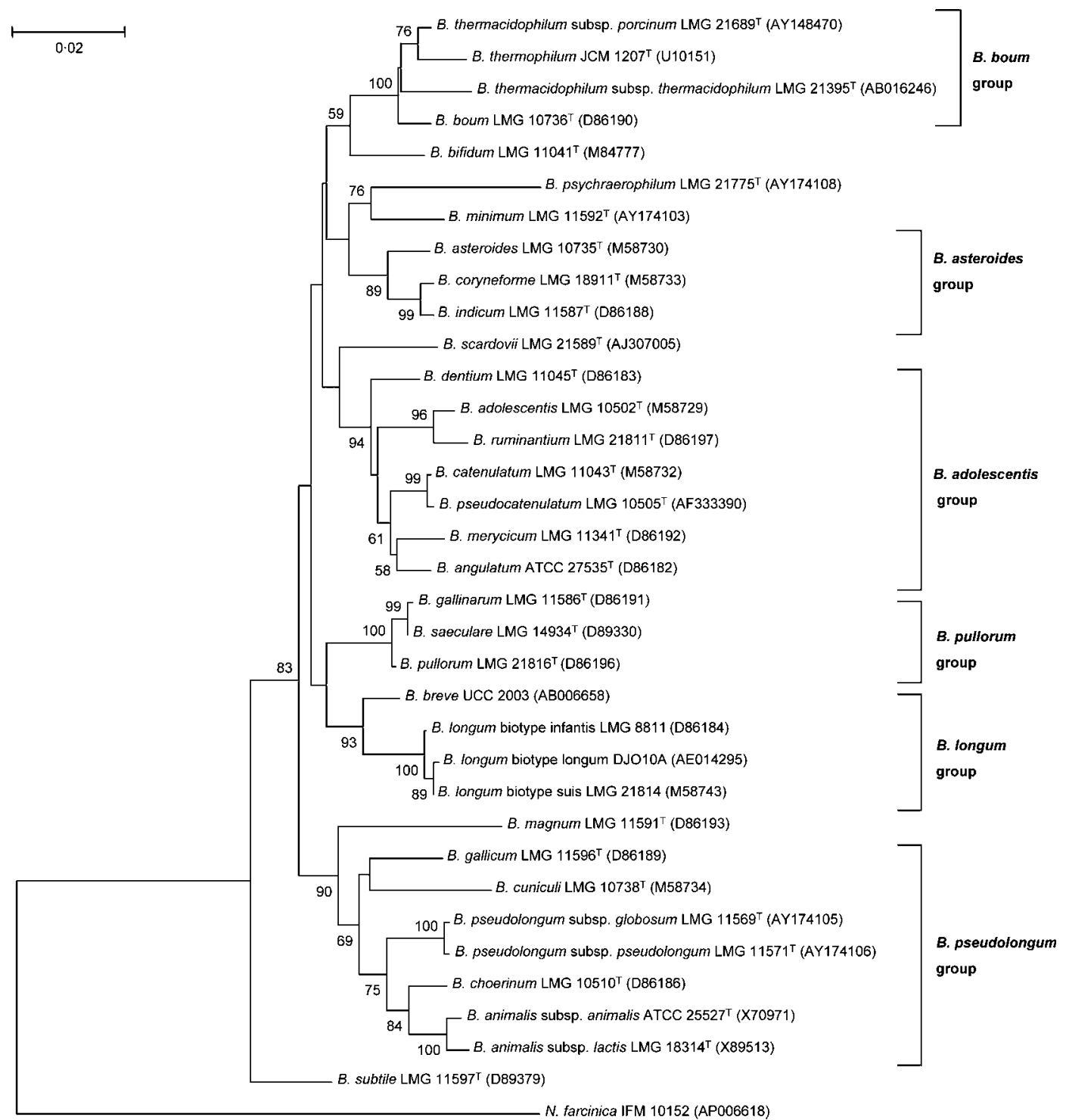

Fig. 1. Phylogenetic tree of the genus Bifidobacterium computed from $16 \mathrm{~S}$ rRNA gene sequences. The tree was rooted using Nocardia farcinica IFM 10152. Bar, 0.02 substitutions per nucleotide position.

Consequently, it was impossible to discriminate subspecies of B. animalis (Ventura et al., 2001b; Masco et al., 2004) or B. longum biotype longum from $B$. longum biotype suis (Ventura et al., 2001a), on the basis of their respective 16S rRNA gene sequences. This led us to use alternative genes, such as the $c l p C, d n a B, d n a G, d n a J 1, p u r F, r p o C$ and $x f p$ genes, which are widely distributed and highly conserved among actinobacteria (C. Canchaya, M. Ventura, G. Fitzgerald and D. van Sinderen, unpublished data). These housekeeping genes have already demonstrated their enormous value as tools for bacterial identification for many other eubacterial taxa (Ludwig \& Schleifer, 1999).

In order to verify the likely relationship between bacterial gene function and bacterial phylogeny, we used housekeeping genes belonging to the main groups of bacterial
Clusters of Orthologous Groups of proteins (COG) functional categories. The $c l p C$ and dnaJ1 genes encode proteins which belong to the chaperone functional category, whereas $d n a B$ and $d n a G$ encode proteins that belong to the replication and repair COG category. The $p u r F, r p o C$ and $x f p$ genes encode proteins belonging to the nucleotide metabolism and transport, transcription and carbohydrate metabolism COG functional categories, respectively.

The $c l p C$ gene encodes a Clp ATPase protein which was previously shown to be induced in response to moderate heat shock regimes in B. breve UCC 2003 (Ventura et al., 2005b). The $c l p C$ sequences were used to calculate a phylogenetic tree including 33 strains. These sequences showed a mean similarity of $81.35 \%$, with a mean SD of $0 \cdot 054$ (Table 2). Each species was differentiated as a distinct 
Table 2. Gene features

Simple mean pairwise distances (p distance) and mean pairwise distances as calculated using Kimura's two parameter model substitution $(K)$ are provided. SD, Standard deviation.

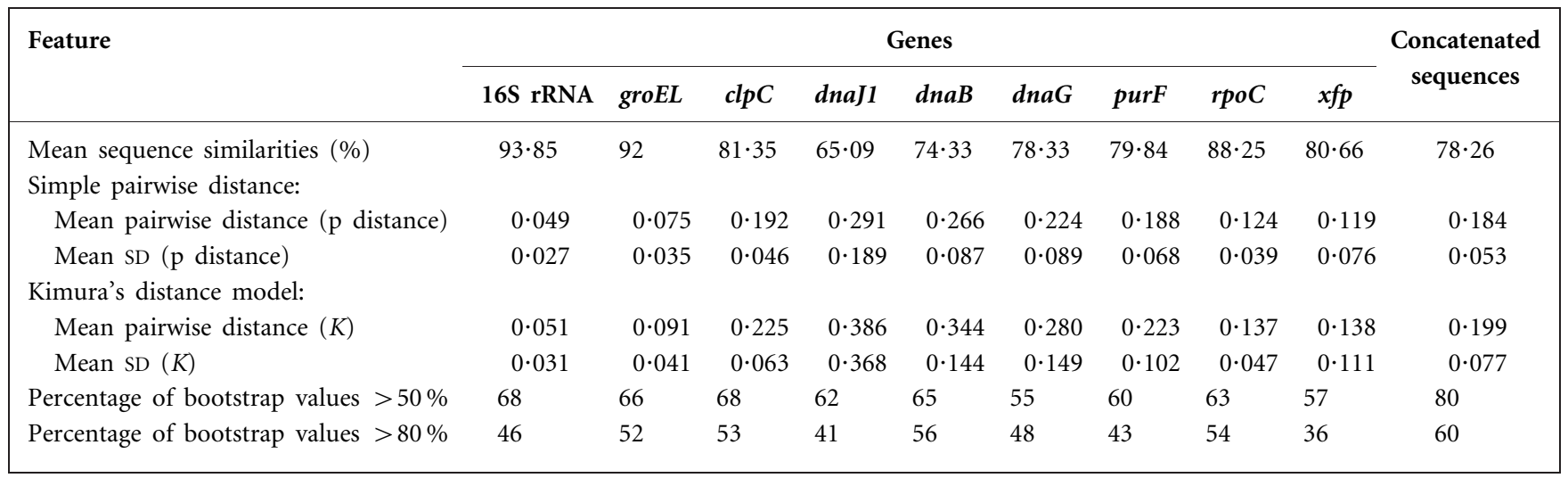

entity in the phylogenetic tree. In this tree, $53 \%$ of the nodes were supported by bootstrap values greater than $80 \%$, indicating that the confidence of the $c l p C$-based tree is high.

Phylogenetic positioning of bifidobacteria species based on $c l p C$ sequences was generally in agreement with that determined by $16 \mathrm{~S}$ rRNA gene sequences (see Supplementary Fig. S1). In fact, the clustering of both trees is very similar, except for a small number of taxa such as Bifidobacterium scardovii or B. bifidum. The pairwise distances between the $c l p C$ sequences were calculated by the maximum-likelihood procedure (Felsenstein, 1989) and compared with the distances between the corresponding $16 \mathrm{~S}$ rRNA gene sequences. Interestingly, the mean pairwise distance of $c l p C$ sequences was $0 \cdot 192$ compared with a mean $16 \mathrm{~S}$ rRNA gene sequence distance of $0 \cdot 049$, indicating that the $c l p C$ gene sequences possess a higher power of discrimination (Table 2).

The DnaJ chaperone is encoded by $d n a J 1$ which is organised with the dnaK, $\operatorname{grp} E$ and $h s p R$ genes in a polycistronic operon in the B. breve UCC 2003 genome (Ventura et al., 2005a). Interestingly, a paralogous gene, dnaJ2, has been found in bifidobacteria (Ventura et al., 2005c). However, sequence analysis, as well as phylogenetic analysis, of $d n a J 1$ and $d n a J 2$ revealed that these genes have followed a separate evolutionary development. The dnaJ1 gene of the Actinobacteria has evolved from an ancient origin that existed before the evolutionary split of the Actinobacteria from the Firmicutes (Ventura et al., 2005c).

The dnaJ1 gene is the most variable gene of this study. From a 537 bp DNA fragment (see Supplementary Fig. S2 in IJSEM Online), we observed a mean rate of similarity close to $65 \%$ at the DNA level in the 31 strains examined (Table 2). This extensive variability may explain the difficulties in obtaining this gene by PCR in some of the bifidobacteria. However, the dnaJ1-based tree showed a relatively limited confidence compared with the other molecular markers studied, since only $41 \%$ of nodes were supported by bootstrap values greater than $80 \%$ (Table 2).

In contrast, the rpoC gene, which encodes a DNA-directed RNA polymerase beta subunit, shows a much higher level of sequence conservation when compared with the other housekeeping genes used in this study. In fact, mean sequence similarity for a $690 \mathrm{bp} r p o C$-fragment is above $88 \cdot 25 \%$ (Table 2 ) and the mean pairwise distance of $r p o C$ sequences was $0 \cdot 130$ compared with the mean $c l p C$ or $d n a J 1$ sequence distance (Table 2). Thus, the discriminatory power of $r p o C$ is lower than that of $c l p C$ or $d n a J 1$, but still stronger than that of $16 \mathrm{~S}$ rRNA gene sequences. Interestingly, several closely related bifidobacterial groups that are not discriminated by $16 \mathrm{~S}$ rRNA gene sequences are resolved well using the $r p o C$ gene sequence (see Supplementary Fig. S3 in IJSEM Online). However, bifidobacterial species such as $B$. breve and $B$. scardovii or $B$. bifidum and B. magnum, which are not closely related on the basis of other molecular markers, carry highly similar $r p o C$ genes.

The purF gene encodes a putative phosphoribosyl pyrophosphate amidotransferase, which catalyses the first committed step in purine biosynthesis. The purF analysis included 33 strains with a sequence length of 450 bp (see Supplementary Fig. S4). These sequences show 79.84\% (Table 2) mean similarity and $43 \%$ of the nodes were supported by bootstrap values greater than $80 \%$ (Table 2). These data, taken together with the fact that the mean pairwise distance of purF gene sequences was $0 \cdot 188$ compared with a mean $16 \mathrm{~S}$ rRNA gene distance of 0.049 and with those of the other molecular markers (Table 2), indicates that the use of this gene as a phylogenetic molecule provides a high discriminatory resolution.

The $d n a B$ and $d n a G$ genes code for a putative replicative DNA helicase and a putative bacterial primase DnaG, respectively. In Escherichia coli, homologous proteins are involved in the initiation of bidirectional replication at the 
origin (oriC), which occurs through the concerted action of the DnaA replication initiator protein, the hexameric DnaB helicase, the DnaC helicase loading partner and the DnaG primase leading to the establishment of two replication forks (Schaeffer et al., 2005). Phylogenetic trees based on dnaB or $d n a G$ sequences are generally in agreement with the $16 \mathrm{~S}$ rRNA gene sequence-derived phylogeny, except for a small number of branching discrepancies (see Supplementary Figs S5 and S6). Interestingly, closely related strains which exhibit nearly identical $16 \mathrm{~S}$ rRNA gene sequences, e.g. $B$. animalis subsp. animalis $-B$. animalis subsp. lactis and $B$. longum biotype longum- $B$. longum biotype infantis- $B$. longum biotype suis species, are clearly branching separately in the trees based on $d n a B$ and $d n a G$ gene sequences (Supplementary Figs S5 and S6). The dnaB and dnaG gene sequences show 74.33 and $78.33 \%$ mean similarity, respectively, while the pairwise distance of the $d n a B$ and dnaG gene sequences is 0.305 and $0 \cdot 252$, respectively (Table 2). This compares favourably to the mean pairwise distances of the 16S rRNA gene and the other molecular markers used in this study (Table 2) and indicates that the $d n a B$ and $d n a G$ sequences produce phylogenetic trees with excellent discriminatory resolution. However, the accuracy of the $d n a G$-based tree is limited because only $48 \%$ of the bootstrap values are greater than $80 \%$ (Table 2).

The $x f p$ gene encodes one of the two F6P-phosphoketolases reported to be present in bifidobacterial genomes (Grill et al., 1995; Meile et al., 2001). The xfp gene is characteristic of the Bifidobacterium genus, although some non-bifidobacterial species have generated amplicons with specific PCR primers designed to amplify an internal $x f p$ region (Yin et al., 2005). For the purpose of this study, we decided to complete the $x f p$ gene sequence database by sequencing the $x f p$ gene of $B$. thermophilum subsp. thermacidophilum and B. thermophilum subsp. porcinum. The tree derived from $x f p$ sequences shows that this gene is able to resolve close related taxa distinctly, i.e. B. animalis subsp. animalis-B. animalis subsp. lactis and $B$. longum biotype longum- $B$. longum biotype infantis- $B$. longum biotype suis species (see Supplementary Fig. S7). Moreover, the high $x f p$-based discriminatory power is also supported by the sequence similarity $(80.66 \%)$ and by bootstrap values greater than $80 \%$ ( $36 \%$ of the nodes).

Generally, all of the genes tested provided enhanced discriminatory powers as compared with the 16S rRNA gene sequences (Table 2). In order to corroborate this important feature, we calculated the pairwise distances between $c l p C, d n a B, d n a G, d n a J 1$, purF, rpoC and $x f p$ gene sequences and compared the values obtained with the distances between the corresponding 16S rRNA gene sequences. The results are presented as a histogram in Fig. 2. The bifidobacterial dnaJ1 sequences were found to be significantly more distant from each other than the $16 \mathrm{~S}$ rRNA gene sequences and other housekeeping gene sequences used in this study. In the dnaJ1 gene distance matrix, there were 23 and $75 \%$ similarities in the range of

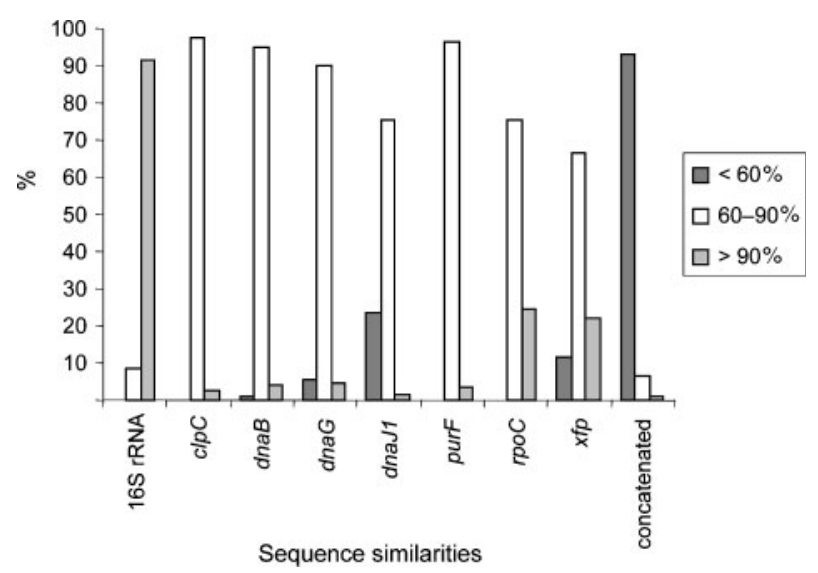

Fig. 2. Histogram showing the distribution of pairwise DNA sequence similarities for the $16 \mathrm{~S}$ rRNA-, clpC-, dnaB-, dnaG-, dnaJ1-, purF-, rpoC- and $x f p$-genes and the concatenated sequences for bifidobacteria.

similarity of less than $60 \%$ and $60-90 \%$, respectively, as compared with $0 \%$ and $8 \%$ for the corresponding similarity classes in the $16 \mathrm{~S}$ rRNA gene distance matrix. For the $16 \mathrm{~S}$ rRNA, rpoC, $x f p$, dnaB, dnaG, dnaJ, purF and $c l p C$ genes, $92 \%, 25 \%, 22 \%, 4 \%, 5 \%, 1 \%, 4 \%, 1 \%$ and $3 \%$ similarities, respectively, are distributed in the similarity class of $>90 \%$, which reinforces the notion that $16 \mathrm{~S}$ rRNA gene sequences are less discriminatory than the alternative sequences used in this study (Fig. 2).

All new data obtained by the use of these alternative molecular markers were compared with another potential molecular marker, i.e. groEL gene. A phylogenetic tree based on this latter molecular marker was constructed using the groEL gene sequences available on public databases (Supplementary Fig. S8). The resulting tree positioning of bifidobacterial species was in agreement with those determined for the $16 \mathrm{~S}$ rRNA sequences, which confirmed previously published data (Ventura et al., 2004c; Jian et al., 2001). Interestingly, the sequence variability of the groEL gene is greater than that of the $16 \mathrm{~S}$ rRNA gene, but significantly less than that of the other molecular markers investigated here (Table 2). Thus, the discriminatory power of groEL is greater than 16S rRNA gene, but still lower than that of the other molecular markers studied here.

\section{Conservation of c/pC, dnaG, dnaJ1 and purF genes across Bifidobacterium genomes}

The $c l p C, d n a G, d n a J 1$ and purF sequences were amplified from 33, 32, 31 and 33 bifidobacterial species, respectively, of the 34 currently described species in the genus Bifidobacterium (Ventura et al., 2004b). In order to determine whether $c l p C, d n a G, d n a J 1$ and purF homologues are present in all bifidobacteria, amplified segments of the $c l p C, d n a G, d n a J 1$, purF genes were used as probes for hybridization to genomic DNA of various bifidobacterial 


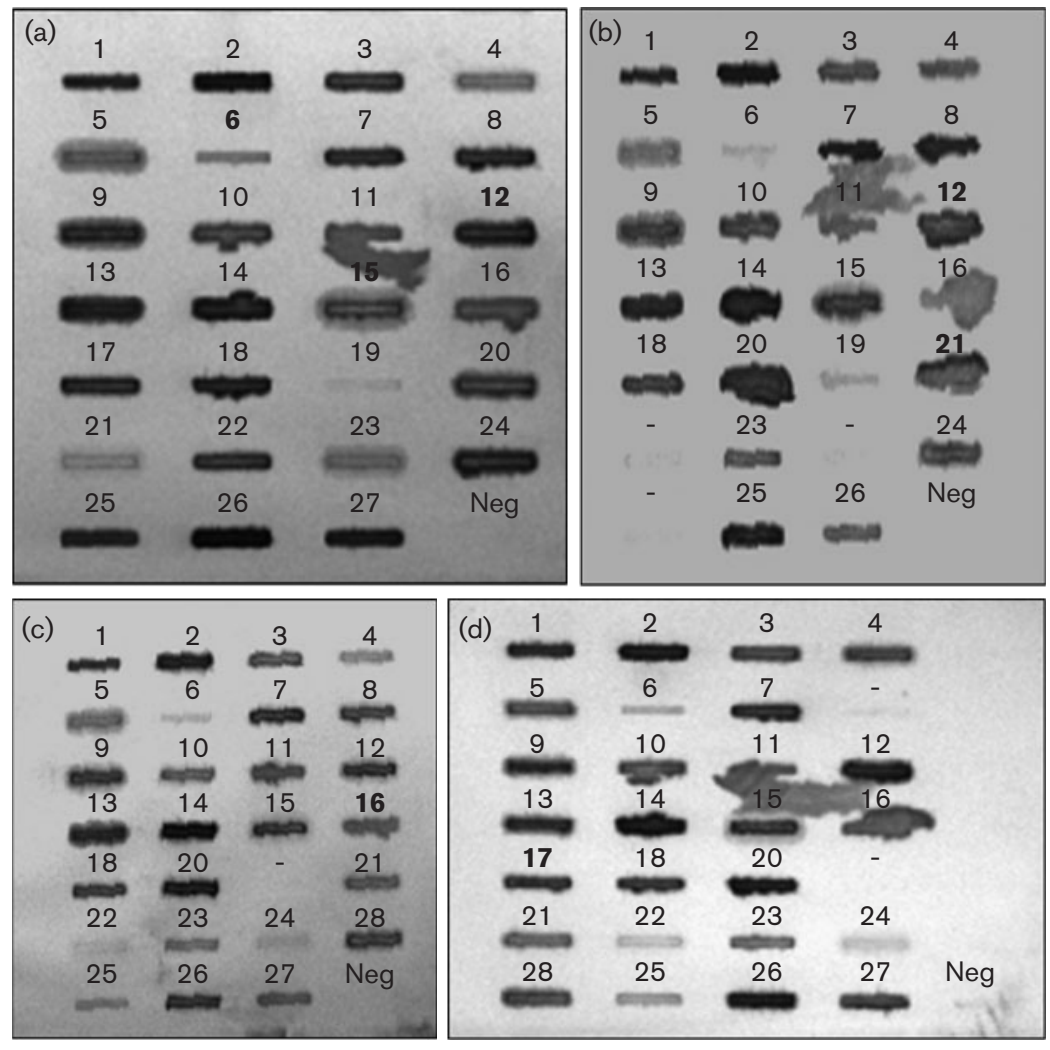

Fig. 3. Slot blot hybridization using DNA extracted from different bifidobacterial species and hybridized using probes for (a) dnaJ1; (b) dnaG; (c) clpC; (d) purF. Letters in bold indicate spots corresponding to a strain that did not give any amplification of the corresponding gene. The order of DNA spotting is as follows: $1, B$. bifidum LMG $11041^{\top} ; 2$, B. subtile LMG $11597^{\top} ; 3, B$. asteroides LMG $10735^{\top} ; 4$, B. magnum LMG $11591^{\top} ; 5, \quad$ B. catenulatum LMG $11043^{\top} ; 6$, B. indicum LMG $11587^{\top} ; 7, B$. pseudolongum subsp. globosum LMG $11569^{\top} ; 8$, B. gallicum LMG $11596^{\top} ; 9, B$. longum biotype suis LMG $21814 ; 10, B$. cuniculi LMG $10738^{\top}$; 11 , B. psychraerophilum LMG $21775^{\top} ; 12, B$. angulatum ATCC $27535^{\top} ; 13, \quad B$. dentium LMG $11045^{\top}$; 14, B. choerinum LMG $10510^{\top}$; 15 , B. merycicum LMG $11341^{\top} ; 16, B$. minimum LMG $11592^{\top} ; 17, B$. saeculare LMG $14934^{\top} ; 18$, B. gallinarum LMG $11586^{\top} ; 19$, B. pullorum LMG $21816^{\top} ; 20$, $B$. animalis subsp. lactis LMG $18314^{\top} ; 21$, $B$. thermacidophilum subsp. thermacidophilum LMG $21395^{\top} ; 22$, B. ruminatium LMG $21811^{\top} ; 23, B$. thermacidophilum subsp. porcinum LMG $21689^{\top} ; 24$, B. pseudocatenulatum LMG $10505^{\top} ; 25$, B. pseudolongum subsp. pseudolongum LMG $11571^{\top}$; 26, B. coryneforme LMG $18911^{\top} ; 27, B$. scardovii LMG $21589^{\top} ; 28$, B. boum LMG $10736^{\top}$.

species, including those which failed to generate a specific amplicon of the specific gene. All investigated bifidobacteria yielded a clear hybridization signal (Fig. 3), clearly indicating that $c l p C$-, dnaG-, dnaJ1- and purF-like genes are present in each bifidobacterial genome. It appears that the relatively large sequence variability occurring among homologues of these genes in various bifidobacteria may be responsible for the failure to amplify these genes.

\section{Concatenated trees}

The concatenation of genes has been shown to be extremely useful in order to infer bacterial phylogeny (Teichmann \& Mitchison, 1999). In this study, the phylogenetic tree resulting from the concatenated sequences of $c l p C, d n a B$, $d n a G, d n a J 1, p u r F, r p o C$ and $x f p$ is presented in Fig. 4. This tree includes the 31 strains for which we were able to sequence the seven genes. The global DNA G $+\mathrm{C}$ content resulting from the concatenation is $63 \%$, which is close to the mean DNA $\mathrm{G}+\mathrm{C}$ content of members of the genus Bifidobacterium.

The discriminatory power of the concatenated tree is much more significant than that observed with the single $16 \mathrm{~S}$ rRNA gene and this is confirmed by the analysis of the pairwise distances and the standard deviation, respectively (Table 2). Each bifidobacterial species was differentiated as a distinct entity. The mean similarity of these concatenated sequences was $78 \cdot 26 \%$ (Table 2) compared with $93 \cdot 85 \%$ with the $16 \mathrm{~S}$ rRNA gene alone and the distance matrix included a smaller number of low values (data not shown). The most closely related taxa exhibited similarities greater than $97 \cdot 8 \%$, i.e., $97 \cdot 8 \%$ for $B$. longum biotype longum and B. longum biotype suis, $95.6 \%$ for $B$. longum biotype longum and $B$. longum biotype infantis, $95.6 \%$ for $B$. animalis subsp. animalis and $B$. animalis subsp. lactis, $94.6 \%$ for $B$. pseudolongum subsp. pseudolongum and $B$. pseudolongum subsp. globosum. These values were lower than those observed for a similar set of strains using $16 \mathrm{~S}$ rRNA gene sequences. Furthermore, the increase in sequence size led to a considerable increase in tree robustness. In fact, the progressive concatenation showed an increase in deep-node bootstrap values. For example, the cluster B. asteroides $-B$. coryneforme, which is supported by bootstrap values of $89,99,96,96,53,90,100,75$ for single $16 \mathrm{~S}$ rRNA, clpC, dnaB, dnaG, dnaJ1, purF, rpoC and $x f p$ genes, respectively, is fully supported in the concatenated tree $(100 \%)$. Data concatenation therefore provides a good means of increasing the robustness of the final tree. This strong increase in bootstrap values demonstrates that a 


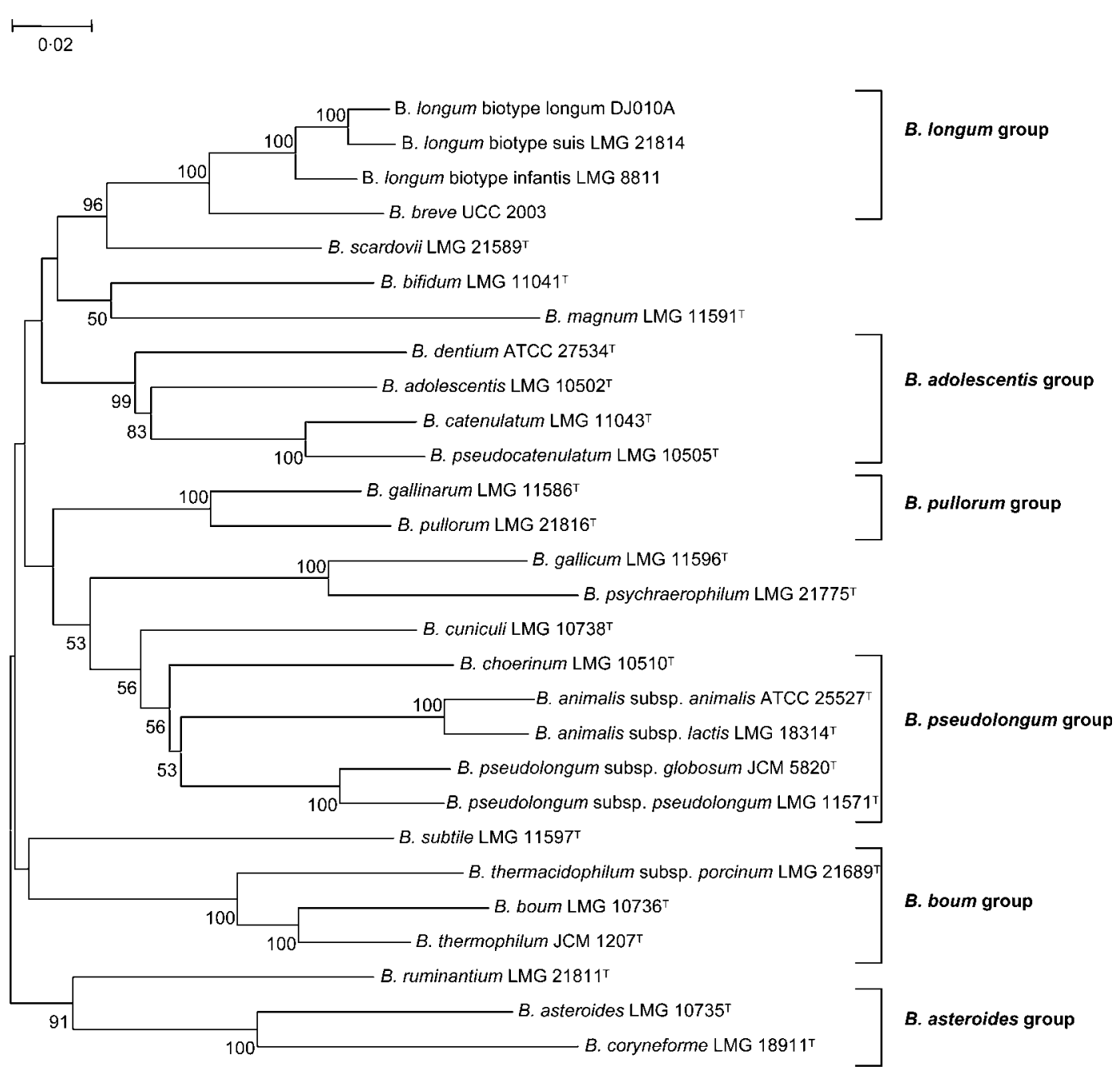

Fig. 4. Phylogenetic tree of the genus Bifidobacterium computed from the concatenation of $c / p C, d n a B, d n a G, d n a J 1, p u r F$, $r p o C$ and $x f p$ gene sequences by the neighbour-joining method and Kimura's two parameter model as the substitution model. Bar, 0.02 substitutions per nucleotide position.

phylogenetic tree calculated from the concatenation of several genes, such as alternative molecular markers to the 16S rRNA gene, may considerably improve the phylogenetic relevance.

The different genes used in this study were selected from different locations on the bifidobacterial genome. However, our phylogenetic analysis points out that they have evolved in the same way. From this multigene concatenation analysis, we have observed that all bifidobacterial groups are derived from an ancestor of the B. asteroides $-B$. coryneforme-B. ruminantium group. In contrast, the speciation of this remnant bifidobacterial taxa has evolved more recently.

From a taxonomical perspective, the DNA-DNA relatedness parameter and $\Delta T_{\mathrm{m}}$ remain the acknowledged standards for species delineation. The phylogenetic definition of species would generally include strains with $70 \%$ or greater
DNA-DNA relatedness and with a $\Delta T_{\mathrm{m}}$ of $5{ }^{\circ} \mathrm{C}$ or less. A bacterial taxon having $70 \%$ or more DNA-DNA relatedness generally has at least $97 \%$ sequence identity in the 16S rRNA gene (Stackebrandt \& Goebel, 1994). The mean distance observed in the bifidobacterial 16S rRNA gene sequences is $5 \%$ and analysis of the distance matrix shows that most closely related species have distances much lower than $3 \%$. As described above, the progressive concatenation of new genes allowed us to significantly reduce the number of small distances and thus increase the discriminatory power. Within the framework of bacterial species identification, the discriminatory capacity of a gene may be defined as its ability to resolve the phylogenetic differences between closely related species. In order to avoid ambiguous identification, it would be interesting to consider a minimal threshold. Indeed, in our study, we used mainly type strains for each bifidobacterial species thus preventing the integration of intraspecific variability. 


\section{Conclusions}

Molecular tools, especially those that are based on the $16 \mathrm{~S}$ rRNA gene, have revolutionized the approaches to determine the taxonomy and evolution of micro-organisms. However, due to the polymorphism and low variability of $16 \mathrm{~S}$ rRNA gene sequences additional molecular markers have been proposed for studying bifidobacterial phylogeny. In this work, we developed a multigene sequence database incorporating seven genes $(c l p C, d n a B, d n a G, d n a J 1, p u r F$, $r p o C$ and $x f p$ ) within the genus Bifidobacterium. This multigenic approach is consistent with the recent recommendations of the ad hoc committee for re-evaluation of the bacterial species definition (Stackebrandt et al., 2002). The concatenation of these seven genes provides a reliable phylogenetic tree which is more discriminatory and robust. In general, concatenation of appropriate sequences prior to tree reconstruction is a useful approach to generate consensus trees. However, concatenated trees do not necessarily provide higher resolution or more reliable phylogenetic relationships and may mask problems of paralogy or xenology. The usefulness of single genes for inferring organismal phylogeny might be sometimes biased by gene duplication, i.e. paralogy, thus some caution should be used in such situations. Paralogy might be involved in the case of rpoC in the B. scardovii-B. breve and B. ruminantium-B. asteroides species or purF in the case of B. gallicumB. gallinarum-B. psychraerophilum, where the apparent high sequence identity appears to be in conflict with the assignment of these strains to different groups in the majority of the other gene trees. Such a suspected case of paralogy seems to affect the tree topology of the concatenated sequence-based tree, where a monophyletic status of $B$. scardovii and the $B$. longum-B. breve cluster is evident and supported by high bootstrap values. Thus, the $B$. scardovii phylogeny is still unresolved. The method of concatenation, however, like all phylogenetic methods, has its strengths and weaknesses. Genes have rich and varied evolutionary histories that are dependent on selective pressures and random events at many different levels. Thus, there may well be genes whose entire evolutionary histories are smooth and regular, but there are surely not enough of these to solve all our phylogenetic questions and we will be forced to use other, locally reliable genes, i.e. $r p o C$, to resolve certain parts of the phylogenetic tree.

Finally, no significant relationship between bacterial gene evolution and gene function has emerged from our analysis, except that different mutation rates may occur between different COG categories. It is noteworthy that those genes involved in DNA replication and DNA repair, e.g. $d n a B$ and $d n a G$, showed high values of mean distances. In contrast, metabolic genes such as $x f p, r p o C$ and $p u r F$ seem to evolve at a lower evolutionary rate. However, such a differential mutation rate between gene categories does not seem to influence the overall evolution of bifidobacterial taxa, but merely affects the mean of divergence between taxa. In this work, we employed mainly type strains (with two exceptions) which does not allow intraspecies variability to be taken into account. However, preliminary sequence intraspecific variability for all seven genes was assessed for a small number of bifidobacterial taxa (e.g. B. longum biotype longum, $B$. dentium and $B$. animalis subsp. lactis). In all these species, the sequence conservation is over $97 \%$ at the DNA level, thus suggesting a high level of conservation of gene sequence at the intraspecies level. Nevertheless, integration of a much larger number of strains of a given species would be required for a better evaluation of the robustness of the multigene approach.

\section{ACKNOWLEDGEMENTS}

This work was financially supported by Enterprise Ireland (grant BR/ 1998/202), by an IRCSET Embark postdoctoral fellowship scheme 2005 to C. C., by the Higher Education Authority Programme for Research in Third Level Institutions, by the Science Foundation Ireland Alimentary Pharmabiotic Centre located at University College Cork and by the Marie Curie Development Host Fellowship (HPMD-200000027) and by the Italian Award for Outstanding Young Researcher entitled "Incentivazione alla mobilità di studiosi stranieri e italiani residenti all' estero" to $\mathrm{M}$. V.

\section{REFERENCES}

Dellaglio, F. \& Felis, G. (2005). Taxonomy of lactobacilli and bifidobacteria. Probiotics and Prebiotics: Scientific Aspects. New Zealand: Caister Academic Press.

Dong, X., Xin, Y., Jian, W., Liu, X. \& Ling, D. (2000). Bifidobacterium thermacidophilum sp. nov., isolated from an anaerobic digester. Int J Syst Evol Microbiol 50, 119-125.

Favier, C. F., Vaughan, E. E., de Vos, W. M. \& Akkermans, A. D. L. (2002). Molecular monitoring of succession of bacterial communities in human neonates. Appl Environ Microbiol 68, 219-226.

Felsenstein, J. (1989). PHYLIP-Phylogeny inference package (version 3.2). Cladistics 5, 164-166.

Felsenstein, J. (1993). PHYLIP (phylogeny inference package), version 3.5c. Department of Genome Sciences, University of Washington, Seattle, USA.

Grill, J. P., Crociani, J. \& Ballongue, J. (1995). Characterization of fructose-6-phosphate phosphoketolases purified from Bifidobacterium species. Curr Microbiol 31, 49-54.

Guindon, S. \& Gascuel, O. (2003). A simple, fast, and accurate algorithm to estimate large phylogenies by maximum likelihood. Syst Biol 52, 696-704.

Jian, W., Zhu, L. \& Dong, X. (2001). New approach to phylogenetic analysis of the genus Bifidobacterium based on partial HSP60 gene sequences. Int J Syst Evol Microbiol 51, 1633-1638.

Kimura, M. (1980). A simple method for estimating evolutionary rates of base substitutions through comparative studies of nucleotide sequences. J Mol Evol 16, 111-120.

Kullen, M. J., Brady, L. J. \& O'Sullivan, D. J. (1997). Evaluation of using a short region of the recA gene for rapid and sensitive speciation of dominant bifidobacteria in the human large intestine. FEMS Microbiol Lett 154, 377-383.

Ludwig, W. \& Schleifer, K. H. (1999). Phylogeny of bacteria beyond the $16 \mathrm{~S}$ rRNA standard. ASM News 65, 752-757.

Masco, E., Ventura, M., Zink, R., Huys, G. \& Swings, J. (2004). Polyphasic taxonomic analysis of Bifidobacterium animalis and Bifidobacterium lactis reveals relatedness at the subspecies level: 
reclassification of Bifidobacterium animalis as Bifidobacterium animalis subsp. animalis subsp. nov. and Bifidobacterium lactis as Bifidobacterium animalis subsp. lactis subsp. nov. Int J Syst Evol Microbiol 54, 1137-1143.

Matsuki, T., Watanabe, K., Tanaka, R., Fukuda, M. \& Oyaizu, H. (1999). Distribution of bifidobacterial species in human intestinal microflora examined with $16 \mathrm{~S}$ rRNA-gene-targeted species-specific primers. Appl Environ Microbiol 65, 4506-4512.

Meile, L., Ludwig, W., Rueger, U., Gut, C., Kaufmann, P., Dasen, G., Wenger, S. \& Teuber, T. (1997). Bifidobacterium lactis sp. nov., a moderately oxygen tolerant species isolated from fermented milk. Syst Appl Microbiol 20, 57-64.

Meile, L., Rohr, L. M., Geissmann, T. A., Herensperger, M. \& Teuber, M. (2001). Characterization of the D-xylulose 5-phosphate/D-fructose 6-phosphate phosphoketolase gene ( $x f p$ ) from Bifidobacterium lactis. J Bacteriol 183, 2929-2936.

Miyake, T., Watanabe, K., Watanabe, T. \& Oyaizu, H. (1998). Phylogenetic analysis of the genus Bifidobacterium and related genera based on 16S rDNA sequences. Microbiol Immunol 42, 661-667.

Ouwehand, A. C., Salminen, S. \& Isolauri, E. (2002). Probiotics: an overview of beneficial effects. Antonie van Leeuwenhoek 82, 279-289.

Perrière, G. \& Gouy, M. (1996). WWW-Query: an on-line retrieval system for biological sequence banks. Biochimie 78, 364-369.

Sambrook, J., Fritsch, E. F. \& Maniatis, T. (2001). Molecular Cloning: a Laboratory Manual, 2nd edn. Cold Spring Harbor, NY: Cold Spring Harbor Laboratory.

Scardovi, V. \& Trovatelli, L. D. (1974). Bifidobacterium animalis (Mitsuoka) comb. nov. and the "minimum" and "subtile" groups of new bifidobacteria found in sewage. Int J Syst Bacteriol 24, 21-28.

Schaeffer, P. M., Headlam, M. J. \& Dixon, N. E. (2005). Proteinprotein interactions in the eubacterial replisome. IUBMB Life 57, $5-12$.

Stackebrandt, E. \& Goebel, B. M. (1994). Taxonomic note: a place for DNA-DNA reassociation and 16S rRNA sequence analysis in the present species definition in bacteriology. Int J Syst Bacteriol 44, 846-849.

Stackebrandt, E., Frederiksen, W., Garrity, G. M. \& 10 other authors (2002). Report of the ad hoc committee for the re-evaluation of the species definition in bacteriology. Int J Syst Evol Microbiol 52, 1043-1047.

Teichmann, S. A. \& Mitchison, G. (1999). Is there a phylogenetic signal in prokaryote proteins? J Mol Evol 49, 98-107.

Thompson, J. D., Higgins, D. G. \& Gibson, T. J. (1994). CLUSTAL W: improving the sensitivity of progressive multiple sequence alignment through sequence weighting, position-specific gap penalties and weight matrix choice. Nucleic Acids Res 22, 4673-4680.

Ventura, M. \& Zink, R. (2003). Comparative sequence analysis of the tuf and $r e c A$ genes and restriction fragment length polymorphism of the internal transcribed spacer region sequences supply additional tools for discriminating Bifidobacterium lactis from Bifidobacterium animalis. Appl Environ Microbiol 69, 7517-7522.

Ventura, M., Elli, M., Reniero, R. \& Zink, R. (2001a). Molecular microbial analysis of Bifidobacterium isolates from different environments by the species-specific amplified ribosomal DNA restriction analysis (ARDRA). FEMS Microbiol Ecol 36, 113-121.

Ventura, M., Reniero, R. \& Zink, R. (2001b). Specific identification and targeted characterization of Bifidobacterium lactis from different environmental isolates by a combined multiplex-PCR approach. Appl Environ Microbiol 67, 2760-2765.

Ventura, M., Canchaya, C., van Sinderen, D., Fitzgerald, G. F. \& Zink, R. (2004a). Bifidobacterium lactis DSM 10140: identification of the atp (atpBEFHAGDC) operon and analysis of its genetic structure, characteristics, and phylogeny. Appl Environ Microbiol 70, 3110-3121.

Ventura, M., van Sinderen, D., Fitzgerald, G. F. \& Zink, R. (2004b). Insights into the taxonomy, genetics and physiology of bifidobacteria. Antonie van Leeuwenhoek 86, 205-223.

Ventura, M., Canchaya, C., Zink, R., Fitzgerald, G. F. \& van Sinderen, D. (2004c). Characterization of the groEL and groES loci in Bifidobacterium breve UCC 2003: genetic, transcriptional and phylogenetic analyses. Appl Environ Microbiol 70, 6197-6209.

Ventura, M., Zink, R., Fitzgerald, G. F. \& van Sinderen, D. (2005a). Gene structure and transcriptional organization of the $d n a \mathrm{~K}$ operon of Bifidobacterium breve UCC 2003 and application of the operon in bifidobacterial tracing. Appl Environ Microbiol 71, 487-500.

Ventura, M., Fitzgerald, G. F. \& van Sinderen, D. (2005b). Genetic and transcriptional organization of the $c l p C$ locus in Bifidobacterium breve UCC 2003. Appl Environ Microbiol 71, 6282-6291.

Ventura, M., Canchaya, C., Bernini, V., Del Casale, A., Dellaglio, F., Neviani, E., Fitzgerald, G. F. \& van Sinderen, D. (2005c). Genetic characterization of the Bifidobacterium breve UCC $2003 \mathrm{hrcA}$ locus. Appl Environ Microbiol 71, 8998-9007.

Yin, X., Chambers, J. R., Barlow, K., Park, A. S. \& Wheatcrof, R. (2005). The gene encoding xylulose-5-phosphate/fructose-6phosphate phosphoketolase $(x f p)$ is conserved among Bifidobacterium species within a more variable region of the genome and both are useful for strain identification. FEMS Microbiol Lett 246, 251-257. 